\title{
A clear urine specimen on visual inspection cannot totally exclude a diagnosis of urinary tract infection
}

Bulloch B, Bausher JC, Pomerantz WJ, et al. Can urine clarity exclude the diagnosis of urinary tract infection? Pediatrics 2000 Nov;106:e60.

\section{QUESTION: Can visual inspection of urine specimen clarity be used to exclude a diagnosis of urinary tract infection (UTI)?}

\section{Design}

Blinded comparison of visual inspection of urine specimen clarity with standard urinalysis (diagnostic standard).

\section{Setting}

The emergency department (ED) of a children's hospital in Cincinnati, Ohio, USA.

\section{Participants}

Convenience sample of 159 previously healthy patients $<21$ years of age (mean age $5.8 \mathrm{y}, 77 \%$ girls) who had either a catheterised $(44 \%)$ or midstream $(56 \%)$ urine specimen collected for urine culture. Exclusion criteria were referral for evaluation of an abnormal urinalysis or positive urine culture, current antibiotic use, an underlying medical problem requiring repeat catheterisation (eg, neurogenic bladder), inability to obtain $3 \mathrm{ml}$ of urine in excess of that required for the laboratory, or underlying renal or genitourinary abnormality.

Description of test and diagnostic standard One investigator, blinded to patient clinical information, visually inspected the samples by holding a standard blood tube with $3 \mathrm{ml}$ of urine $1 \mathrm{~cm}$ from a standard white background with black printed 11 font text under normal fluorescent lighting. Another tube with water was viewed under the same conditions. If the printed text was as legible through the urine as through the water, the sample was considered to be clear. If the urine was not clear, acetic acid solution was added to dissolve any phosphates, and the visual inspection was repeated. A second observer blinded to clinical information and the findings of the first observer, did the same visual inspection. If the 2 observers disagreed, the specimen was considered cloudy. The diagnostic standard of laboratory urinalysis included dipstick testing for presence of nitrites and leukocyte esterase, bacteria on microscopy, and white cell count (per high powered field).

\section{Main outcome measures}

Sensitivity, specificity, and positive and negative likelihood ratios (LRs).

\section{Main results}

$18 \%$ of urine samples had positive cultures $(76 \%$ of these samples were positive for Escherichia coli). Girls accounted for $83 \%$ of positive cultures. The kappa for agreement between observers was 0.88 . The table summarises the test characteristics for visual inspection.

\section{Conclusion}

A clear urine specimen on visual inspection cannot totally exclude a diagnosis of urinary tract infection. diagnosis of urinary tract infection*

${ }^{*} \mathrm{LR}=$ likelihood ratio. Diagnostic terms defined in glossary.

\section{COMMENTARY} less than 1 in 100. of the test. clarity can best be used to reliably exclude a diagnosis of UTI. Williams and Wilkins, 1999:1560-1. nary tract infection. Arch Pediatr Adolesc Med 2000;154:386-90.
Source of funding: no
external funding.

For correspondence: Dr B Bulloch, Children's Hospital, 820 Sherbrook Street, Room 208A, Winnipeg, Manitoba R3A 1S1, Canada.Fax $+12047874807$

Test characteristics of visual inspection of urine specimen clarity (clear) for exclusion of a

\begin{tabular}{lllll}
$\begin{array}{l}\text { Method of specimen } \\
\text { collection }\end{array}$ & Sensitivity (95\% CI) & Specificity (CI) & +LR & -LR \\
\hline All specimens & $90 \%(73$ to 98$)$ & $82 \%(76$ to 89$)$ & 5.07 & 0.13 \\
\hline Catheterised specimens & $83 \%(52$ to 98$)$ & $86 \%(75$ to 94$)$ & 6.04 & 0.19 \\
\hline Midstream specimens & $94 \%(71$ to 100$)$ & $79 \%(68$ to 88$)$ & 4.52 & 0.07 \\
\hline
\end{tabular}

Bulloch $e t$ al have posed an intriguing question about the usefulness of a urine clarity test to exclude a diagnosis of UTI. For children, UTIs rank second only to upper respiratory infections as a source of morbidity from bacterial infection. ${ }^{1}$ The study aimed to find a way to exclude UTI by checking urine clarity and showed the potential for this simple test. Based on a negative LR of 0.13 and a pretest probability of UTI of 1 out of 20, a clear urine sample may lower the likelihood of a UTI to a post-test probability of

How applicable is this study to practice? The authors have clearly described the test, a simple process of reading text through a urine sample, which achieved a high level of agreement. The study sample was described briefly as a convenience sample, with patients being enrolled only when 1 of the investigators was in the ED. No information was given about the ED's overall population of eligible participants during the study period. More information about this may have explained the study's high prevalence rate of $18 \%$ in contrast to a cross sectional study that reported an overall prevalence of $3.3 \%$ for UTI in children younger than 2 years presenting to the ED with fever of an unknown source. ${ }^{2}$ Furthermore, a convenience sample may not include a broad enough spectrum of disease presentation, possibly leading to a misestimation of the usefulness

Overall, the usefulness of urine clarity to exclude a diagnosis of UTI should be studied further. Clinical decision rules, such as Gorelick and Shaw's model to identify young girls at risk for UTI, ${ }^{3}$ could explore the addition of urine clarity to the model. It is also important to ascertain under which conditions (eg, presenting to the ED with fever or to a primary care setting with symptoms) and for which children (eg, age and race) urine

Gene Elizabeth Harkless, RN, DNSc, ARNP Associate Professor, Department of Nursing University of New Hampshire Durham, New Hampshire, USA

1 Gonzalez ET, Roth DR. Urinary tract infection. In: McMillan JA, DeAngelis CD, Feigin RD, et al, editors. Oski's pediatrics:principles and practice. Third edition. Philadelphia: Lippincott

2 Shaw KN, Gorelick M, McGowan KL, et al. Prevalence of urinary tract infection in febrile young children in the emergency department. Pediatrics 1998;102:e16.

3 Gorelick MH, Shaw KN. Clinical decision rule to identify febrile young girls at risk for uri- 